\title{
Nonlocal Parabolic Integro-differential Equations with Delays
}

\author{
${ }^{1}$ Kamalendra Kumar, ${ }^{2}$ Rakesh Kumar, ${ }^{3}$ R.K. Shukla \\ ${ }^{1}$ Dept. of Mathematics, SRMS CET Bareilly-243 001, India \\ Email: kamlendra.14kumar@gmail.com \\ ${ }^{2}$ Dept. of Mathematics, Hindu College, Moradabad - 244 001, India \\ Email: rakeshnaini@yahoo.co.in \\ ${ }^{3}$ Dept. of Mathematics, Invertis University, Bareilly -243 123, India \\ Email: rkshukla30@gmail.com
}

\begin{abstract}
In this paper we consider a parabolic integro-differential equation with delay and a nonlocal boundary condition. We apply the method of semidiscretization in time, also known as the method of lines, to establish the existence and uniqueness of the considered problem. We also establish the continuous dependence of the solution on the initial data. Finally, an application of the established results is demonstrated.
\end{abstract}

Keywords: Integro-differential equation, weak solution, method of lines

Mathematics Subject Classification (2000): 34K30, 34G20, 47H06, 35K55

\section{Introduction}

In certain physical situation we need to consider more complex type of initial boundary conditions which are different from the traditional type of such conditions. Such type of conditions is termed as nonlocal since these conditions involve several points or a part or the whole domain. We will consider abstract formulations of such type of problems and apply the method of time discretization to establish well-posedness.

In this paper we consider the following parabolic integro-differential equation with delay and a nonlocal boundary condition, 


$$
\frac{\partial u}{\partial t}(x, t)-\frac{\partial^{2} u}{\partial x^{2}}(x, t)=K(u)(t)+f(x, t, u(x, t-\tau)), \tau>0,(x, t) \in(0,1) \times[0, T],
$$

subject to

$$
\begin{aligned}
& u(x, t)=u_{0}(x, t), \quad(x, t) \in(0,1) \times[-\tau, 0], \\
& \frac{\partial u}{\partial x}(0, t)=0, \quad t \in[-\tau, T], \\
& \int_{0}^{1} u(x, t) d x=0, \quad(x, t) \in(0,1) \times[-\tau, T],
\end{aligned}
$$

where $\mathrm{K}$ is a nonlinear Volterra operator given by

$$
K(u)(t)=\int_{0}^{t} k(t-s) g(s, u(s)) d s,
$$

in which $k$ is a real-valued function defined on $J:=[0, T]$ and the map $g$ is defined from $J \times \mathbb{R}$ into $\mathbb{R}, f$ is defined from $(0,1) \times[0, T] \times \mathbb{R}$ into $\mathbb{R}$. The motivation for such problems comes from different branches such as physics, rheology and especially the theory of heat conduction in materials when the inner heat sources are of special types. For example, the hydrational heat, where the intensity of heat sources depends on the amount of heat already developed.

Cannon and Van Der Hoek [6] have studied the heat diffusion equation with an integral boundary condition and have established the existence, uniqueness and continuous dependence on the data. The authors derive an equivalent Volterra integral equation of the second kind and treat the problem numerically. In [7] author has used the Galerkin method and analyzed the numerical solution of the heat equation with an integral condition.

Boundary value problems with integral boundary conditions are an interesting and important class of problems. The starting work on the use of nonlocal boundary conditions has been done by Cannon [5]. Subsequently, similar studies have been done by Kamynin [9], Ionkin [11]. Beilin [15] has considered the wave equation with an integral condition using the method of separation of variables and Fourier series. The study of boundary value problems with integral boundary conditions can be found in the papers $[3,12,13,14,5,6,7]$ and the references given in these papers.

Pulkina [10] has dealt with a hyperbolic problem with two integral conditions and has established the existence and uniqueness of generalized solutions using the fixed point arguments.

The motivation for considering a nonlocal boundary value problem of the type (1.1)-(1.4) comes from the works of $[12,13,14]$. Problem (1.1)-(1.4) with $k=0$ has been considered by Merazga and Bouziani [14] where the authors have considered the two-dimensional diffusion equation and have transformed it into a one-dimensional problem. In $[12,13]$ authors study a linear heat equation and a semilinear heat equation with two integral boundary conditions. Recently DaoQuing and $\mathrm{Yu}$ [3] have established the well-posedness for a semilinear heat equation with integral conditions, on the basis of the solution of a Dirichlet problem for a parabolic equation and a Volterra integral equation. Bahuguna and 
Raghavendra [4] have established the existence and uniqueness of a strong solution using Rothe's method of the following parabolic integro-differential equation

$$
\begin{aligned}
\frac{d u}{d t}(t)+A u(t) & =\int_{0}^{t} a(t-s) k(s, u(s)) d s+f(t, u(t-\tau)), \quad t \in(0, T], \\
u(t) & =u_{0}(t), \quad t \in[-\tau, 0]
\end{aligned}
$$

where $A$ is a nonlinear single-valued operator with the domain and the range in the Banach space whose dual is uniformly convex. The mapping $k$ satisfies a growth condition, functions $f$ and $a$ are continuous having bounded variations on $[0, T]$.

We plan to apply Rothe's method to establish the existence and uniqueness result for (1.1)-(1.4). Rothe's method, also known as the method of lines, is a powerful tool for proving the existence and uniqueness of solutions to evolution equations. This method is oriented towards the numerical approximations. For instance, we refer to Rektorys [8] for a rich illustration of method applied to various interesting physical problems. Bahuguna and Dabas $[1,2]$ have used the method of semidiscretization in time to study a partial differential equation with an integral condition involving delay arising in population dynamics.

\section{Preliminaries and Main Result}

The problem (1.1)-(1.4) is considered in the real Hilbert space $\boldsymbol{H}=L^{2}(0,1)$ of square-integrable functions defined from $(0,1)$ into $\mathbb{R}$ with the inner product and the corresponding norm

$$
(u, v)=\int_{0}^{1} u(x) v(x) d x, \quad\|u\|^{2}=\int_{0}^{1}|u(x)|^{2} d x \quad u, v \in \boldsymbol{H}
$$

Given a function $h:(0,1) \times[a, b] \rightarrow \mathbb{R}$ such that for each $t \in[a, b], h(., t):[a, b] \rightarrow \boldsymbol{H}$, we may identify it with the function $h:[a, b] \rightarrow \boldsymbol{H}$ given by $h(t)(x)=h(x, t)$. The integral condition (1.4) is adjoined with the space itself by taking $\boldsymbol{V} \subset \boldsymbol{H}$ defined by

$$
\boldsymbol{V}=\left\{u \in \boldsymbol{H}: \int_{0}^{1} u(x) d x=0\right\}
$$

$\mathbf{V}$ is closed subspace of $\mathbf{H}$ and hence is a Hilbert space itself with the inner product(.,.), and the corresponding norm $\|$.$\| . By \boldsymbol{H}^{2}(\mathbf{0}, \mathbf{1})$ we denote the usual second order Sobolev space on $(0,1)$. For any Banach space $X$, with the norm $\|$. $\|_{X}$ and an interval $I=[a, b],-\infty<a<b<\infty$, we shall denote $C(I ; X)$ the space of all continuous functions $u$ from $[a, b]$ into $X$ with the norm 


$$
\|u\|_{C(I ; X)}=\max _{a \leq t \leq b}\|u(t)\|_{X}
$$

The space $L^{2}(I ; X)$ consists of all square-Bochner integrable functions (equivalent classes) $u$ such that with the norm

$$
\|u\|_{L^{2}(I ; X)}^{2}=\int_{a}^{b}\|u(t)\|_{X}^{2} d t
$$

Also, we need the space $B_{2}^{1}(0,1)$ introduced by Merazga and A. Bouziani [12] being the completion of the space $C_{0}(0,1)$ of all real continuous functions having compact supports in $(0,1)$ with the inner product and the corresponding norm

$$
(u, v)_{B_{2}^{1}}=\int_{0}^{1}\left(\int_{0}^{x} u(\xi) d \xi\right)\left(\int_{0}^{x} u(\xi) d \xi\right) d x, \quad\|u\|_{B_{2}^{1}}^{2}=\int_{0}^{1}\left(\int_{0}^{x} u(\xi) d \xi\right)^{2} d x
$$

It is clear that $v \in B_{2}^{1}(0,1)$ if and only if $\int_{0}^{x} v(\xi) d \xi \in L^{2}(0,1)$. It follows that the following inequality $\|v\|_{B_{2}^{1}}^{2} \leq \frac{1}{2}\|v\|^{2}$ holds for every $v \in L^{2}(0,1)$, and the embedding $L^{2}(0,1) \rightarrow B_{2}^{1}(0,1)$ is continuous.

We assume the following:

(D1) The function $g$ is continuous in both the variable and for all $t \in J$ and $x \in H$

$$
\|g(t, x)\|_{B_{2}^{1}} \leq C_{1}\|x\|_{B_{2}^{1}}+C_{2},
$$

where $C_{1}, C_{2}$ are positive constants.

(D2) For a.e. $t \in J$ and all $x, y \in H$

$$
\|g(t, x)-g(t, y)\|_{B_{2}^{1}} \leq L(t)\|x-y\|_{B_{2}^{1}}
$$

where $L \in L^{1}(J)$ is nonnegative.

(D3) $u_{0}(x) \in H^{2}(0,1)$ also $u_{0}(x) \in V$, i.e. $\int_{0}^{1} u_{0}(x) d x=0$.

(D4) Functions $f: J \times H \times H \rightarrow H$. The functions $f$ and $k$ are Lipchitz continuous on $J$.

The following defines a weak solution to (1.1)-(1.4).

Definition 2.1 A weak solution $u$ of (1.1)-(1.4) is a function $u(x, t)$ defined on $0 \leq x \leq 1,0 \leq t \leq T$ which has the following properties

$$
\begin{aligned}
& u \in L^{2}(J ; V) \cap C\left(J ; B_{2}^{1}(0,1)\right) \\
& u^{\prime} \in L^{2}\left(J ; B_{2}^{1}(0,1)\right) ; \\
& u(t)=u_{0}(t) \in C\left([-\tau, 0], B_{2}^{1}(0,1)\right)
\end{aligned}
$$




$$
\begin{aligned}
& \int_{0}^{T}\left(v(t), \frac{d u(t)}{d t}\right)_{B_{2}^{1}} d t+\int_{0}^{T}(v(t), u(t)) d t \\
& =\int_{0}^{T}(v(t), K(u)(t)+f(t, u(t-\tau)))_{B_{2}^{1}}, \quad \forall v \in L^{2}(J ; V)
\end{aligned}
$$

Theorem 2.2 Suppose that (D1)-(D4) hold on $J=[0, \tau]$, i.e., $T=\tau$. Then (1.1)(1.4) has a unique weak solution on $J$. For the set of data $\left(u_{i}^{0}, f_{i}\right)$, the corresponding solutions $u_{i}, i=1,2$, satisfy the following estimate

$$
\begin{aligned}
\| u_{1} & -u_{2}\left\|_{C\left(J_{t}, B_{2}^{1}\right)}^{2}+2 \int_{0}^{t}\right\| u_{1}-u_{2} \|_{C\left(J_{s}, L^{2}\right)}^{2} d s \\
& \leq\left(\left\|u_{1}^{0}-u_{2}^{0}\right\|_{B_{2}^{1}}^{2}+C_{T}\left\|u_{1}-u_{2}\right\|_{C\left(J_{t}, B_{2}^{1}\right)}^{2}+\int_{0}^{t}\left\|\tilde{f}_{1}-\tilde{f}_{2}\right\|_{C\left(J_{s}, B_{2}^{1}\right)}^{2} d s\right) \exp \{t\},
\end{aligned}
$$

where $J_{t}=[0, t], 0 \leq t \leq \tau$, and $\tilde{f}_{i}(t)=f_{i}\left(t, u_{0}(t-\tau)\right), i=1,2$. This shows the continuous dependence of the solutions on the data.

In the next section we establish the existence, uniqueness of a weak solution to (1.1)-(1.4). We shall prove theorem 2.2 in the last section with the help of several lemmas.

\section{Discretization Scheme and A Priori Estimates}

Let $t_{j}^{n}$ denote a partition of the interval $J=[0, \tau]$ defined by $t_{j}^{n}=j h_{n}, j=$ $0,1,2, \ldots ., n$, where $h_{n}=\frac{\tau}{n}$, for $n=1,2, \ldots$. We set $u_{0}^{n}=u_{0}$ for all $n \in \mathbb{N}$, and replace, at the point of division $t_{j}^{n}$ the derivative $\frac{\partial u}{\partial t}$ by the corresponding difference quotient and the integral by a sum. In this way, problem (1.1)-(1.4) reduced to the solutions of $n$ elliptic problems.

$$
\begin{aligned}
& \frac{u_{j}^{n}-u_{j-1}^{n}}{h_{n}}-\frac{d^{2} u_{j}^{n}}{d x^{2}}=f_{j}^{n}+h_{n} \sum_{i=0}^{j-1} k\left(t_{j}^{n}-t_{i}^{n}\right) g\left(t_{i}^{n}, u_{i}^{n}\right), \quad x \in(0,1), \\
& \frac{d u_{j}^{n}}{d x}(0) d x=0, \\
& \int_{0}^{1} u_{j}^{n}(x) d x=0,
\end{aligned}
$$

for $j=1,2, \ldots \ldots, n$. We shall denote, for $0 \leq i, j \leq n$, by

$$
\delta u_{j}^{n}=\frac{u_{j}^{n}-u_{j-1}^{n}}{h_{n}},
$$




$$
\begin{aligned}
& f_{j}^{n}=f\left(t_{j}^{n}, u_{0}\left(t_{j}^{n}-\tau\right)\right), \\
& k_{j i}^{n}=k\left(t_{j}^{n}-t_{i}^{n}\right)
\end{aligned}
$$

and

$g_{j}^{n}=g\left(t_{j}^{n}, u_{j}^{n}\right)$

The existence of a unique $u_{j}^{n} \in \boldsymbol{H}^{2}(0,1)$ satisfying (3.11)-(3.13) is ensured as established in theorem 3.2 of [8].

Definition 3.1 Define the "Rothe sequence" $\left\{U^{n}(t)\right\}$ of functions from $J$ into $\boldsymbol{H}^{2}(0,1)$ defined by

$$
U^{n}(t)=u_{j-1}^{n}+\frac{1}{h_{n}}\left(t-t_{j-1}^{n}\right)\left(u_{j}^{n}-u_{j-1}^{n}\right), \quad t \in\left[t_{j-1}^{n}, t_{j}^{n}\right],
$$

for $j=1,2, \ldots \ldots \ldots, n$. Further, we define the sequence $\left\{X^{n}(t)\right\}$ of step functions from $J$ into $\boldsymbol{H}^{2}(0,1)$ by

$$
X^{n}(0)=u_{0}, \quad X^{n}(t)=u_{j}^{n}, \quad t \in\left(t_{j-1}^{n}, t_{j}^{n}\right], \quad j=1,2, \ldots \ldots, n .
$$

We prove the convergence of the sequence $\left\{U^{n}\right\}$ to the unique solution of the problem as $n \rightarrow \infty$ using some a priori estimates on $U^{n}$. We first prove the estimates for $u_{j}^{n}$ and $\delta u_{j}^{n}$ using (D1)-(D4). To establish the estimates first we take the variational formulations of the discretized problem (3.11)-(3.13). For any $v$ be in $V$, we have

$$
\left(v, \delta u_{j}^{n}\right)_{B_{2}^{1}}-\left(v, \frac{d^{2} u_{j}^{n}}{d x^{2}}\right)_{B_{2}^{1}}=\left(v, f_{j}^{n}\right)_{B_{2}^{1}}+\left(v, h_{n} \sum_{i=0}^{j} k_{j i}^{n} g_{i}\right)_{B_{2}^{1}}
$$

Using (3.12), the second term in left hand side of (3.20) become

$$
\begin{aligned}
\left(v, \frac{d^{2} u_{j}^{n}}{d x^{2}}\right)_{B_{2}^{1}} & =\int_{0}^{1}\left[\frac{d u_{j}^{n}}{d x}(x)-\frac{d u_{j}^{n}}{d x}(0)\right]\left(\int_{0}^{x} v(\xi) d \xi\right) d x \\
& =\int_{0}^{1} \frac{d u_{j}^{n}}{d x}(x)\left(\int_{0}^{x} v(\xi) d \xi\right) d x \\
& =\left.u_{j}^{n}(x)\left(\int_{0}^{x} v(\xi) d \xi\right)\right|_{x=0} ^{x=1}-\int_{0}^{1} u_{j}^{n}(x) v(x) d x \\
& =-\left(v, u_{j}^{n}\right) .
\end{aligned}
$$

Finally (3.20) becomes, 


$$
\left(v, \delta u_{j}^{n}\right)_{B_{2}^{1}}+\left(v, u_{j}^{n}\right)=\left(v, f_{j}^{n}\right)_{B_{2}^{1}}+\left(v, h_{n} \sum_{i=0}^{j-1} k_{j i}^{n} g_{i}\right)_{B_{2}^{1}}
$$

In the next lemma we find the estimates by using variational inequality (3.22). Throughout, $C$ will represent a generic constant independent of $j, h_{n}$ and $n$ and $C T, C e^{C T}$, etc., are again replaced by $C$.

Lemma 3.2 Assume the hypothesis (D1)-(D4) are satisfied then there exists a positive constant $C$, independent of $j, h_{n}$ and $n$ such that.

$$
\begin{aligned}
& \left\|u_{j}^{n}\right\|_{\leq C} \leq C \\
& \left\|\delta u_{j}^{n}\right\|_{B_{2}^{1}} \leq C .
\end{aligned}
$$

$n \geq 1$ and $j=1,2, \ldots \ldots, n$.

Proof. In (3.22) let $j=1$ and $v=\delta u_{1}^{n}$, we have

$$
\left(\delta u_{1}^{n}, \delta u_{1}^{n}\right)_{B_{2}^{1}}+h_{n}\left(\delta u_{1}^{n}, \delta u_{1}^{n}\right)=\left(\delta u_{1}^{n}, h_{n} k_{10}^{n} g_{1}^{n}+f_{1}^{n}\right)_{B_{2}^{1}}-\left(\delta u_{1}^{n}, u_{0}^{n}\right) \text {. }
$$

From relation (3.21), we have

$$
\left(\delta u_{1}^{n}, u_{0}^{n}\right)=-\left(\delta u_{1}^{n}, \frac{d^{2} u_{0}}{d x^{2}}\right)_{B_{2}^{1}}
$$

Hence, from (3.25), we have the estimate

$$
\left\|\delta u_{1}^{n}\right\|_{B_{2}^{1}} \leq h_{n}\left|k_{10}^{n}\right|\left\|g_{1}\right\|_{B_{2}^{1}}+\left\|f_{1}^{n}\right\|_{B_{2}^{1}}+\left\|\frac{d^{2} u_{0}^{n}}{d x^{2}}\right\|_{B_{2}^{1}}:=C .
$$

Using identity (3.22) for $j$ and $j-1$, by subtraction, we obtain

$$
\begin{aligned}
\left(\delta u_{j}^{n}, \delta u_{j}^{n}\right)_{B_{2}^{1}} & +h\left(\delta u_{j}^{n}, \delta u_{j}^{n}\right) \\
& =\left(\delta u_{j}^{n}, \delta u_{j-1}^{n}\right)_{B_{2}^{1}}+h_{n}\left(\delta u_{j}^{n}, k_{j j-1}^{n} g_{j-1}^{n}\right)_{B_{2}^{1}} \\
& +h_{n}\left(\delta u_{j}^{n}, \sum_{i=0}^{j-2}\left[k_{j i}^{n}-k_{j-1 i}^{n}\right] g_{i}^{n}\right)_{B_{2}^{1}}+\left(\delta u_{j}^{n}, f_{j}^{n}-f_{j-1}^{n}\right)_{B_{2}^{1}} .
\end{aligned}
$$

Taking the norm, we get

$$
\left\|\delta u_{j}^{n}\right\|_{B_{2}^{1}} \leq\left\|\delta u_{j-1}^{n}\right\|_{B_{2}^{1}}+h_{n}\left|k_{j j-1}\right|\left\|g_{j-1}^{n}\right\|_{B_{2}^{1}}
$$




$$
+h_{n} \sum_{i=0}^{j-2}\left|k_{j i}-k_{j-1 i}\right|\left\|g_{i}^{n}\right\|_{B_{2}^{1}}+\left\|f_{j}^{n}-f_{j-1}^{n}\right\|_{B_{2}^{1}} .
$$

Repeating the inequality (3.27), leads to the estimate

$$
\begin{aligned}
\left\|\delta u_{j}^{n}\right\|_{B_{2}^{1}} \leq\left\|\delta u_{1}^{n}\right\|_{B_{2}^{1}}+h_{n} \sum_{i=1}^{j}\left|k_{i i-1}\right|\left\|g_{i-1}^{n}\right\|_{B_{2}^{1}} \\
+h_{n} \sum_{i=0}^{j-2}\left[\sum_{p=1}^{i}\left|k_{p i}-k_{p-1 i}\right|\right]\left\|g_{i}^{n}\right\|_{B_{2}^{1}}+\sum_{i=1}^{j}\left\|f_{i}^{n}-f_{i-1}^{n}\right\|_{B_{2}^{1}} .
\end{aligned}
$$

Making use of (D1) and (D4) in inequality (3.28), we obtain

$$
\begin{aligned}
\left\|\delta u_{j}^{n}\right\|_{B_{2}^{1}} \leq \| & \delta u_{1}^{n} \|_{B_{2}^{1}} \\
& +k_{T} h_{n} \sum_{i=1}^{j}\left(C_{1}\left\|u_{i-1}^{n}\right\|_{B_{2}^{1}}+C_{2}\right)+V_{T}(k) h_{n} \sum_{i=0}^{j-2}\left(C_{1}\left\|u_{i}^{n}\right\|_{B_{2}^{1}}+C_{2}\right) \\
& +V_{T}(f)
\end{aligned}
$$

where $k_{T}=\sup _{t \in J}|k(t)|$ and $V_{T}(k), V_{T}(f)$ are the total variations of $k$ and $f$ respectively. Using the fact that $(j-1) h_{n} \leq T$ and all constant term by generic positive constant $C$, we have

$$
\begin{aligned}
\left\|\delta u_{j}^{n}\right\|_{B_{2}^{1}} \leq C h_{n} & \sum_{i=0}^{j-1}\left\|u_{i}^{n}\right\|_{B_{2}^{1}}+C \\
& \leq C h_{n} \sum_{i=0}^{j-1}\left(h_{n} \sum_{r=1}^{i}\left\|\delta u_{i}^{n}\right\|_{B_{2}^{1}}+\left\|u_{0}\right\|_{B_{2}^{1}}\right)+C \\
& \leq C h_{n} \sum_{i=1}^{j-1}\left\|\delta u_{i}\right\|_{B_{2}^{1}}+C,
\end{aligned}
$$

Using the discrete version of Gronwall's inequality (see Rektorys [8], Remark 15.3 , p. 286) in (3.29) we obtain the desired estimate (3.24) of the lemma. Now taking $v=u_{j}^{n}-u_{j-1}^{n}$ in (3.22) and using the identity, 
we get

$$
\left(u_{j}^{n}, u_{j}^{n}-u_{j-1}^{n}\right)=\frac{1}{2}\left(\left\|u_{j}^{n}-u_{j-1}^{n}\right\|^{2}+\left\|u_{j}^{n}\right\|^{2}-\left\|u_{j-1}^{n}\right\|^{2}\right)
$$

$$
\begin{aligned}
h_{n}\left\|\delta u_{j}^{n}\right\|_{B_{2}^{1}}^{2}+ & \frac{1}{2}\left\|u_{j}^{n}-u_{j-1}^{n}\right\|^{2}+\frac{1}{2}\left\|u_{j}^{n}\right\|^{2} \\
& =\left(u_{j}^{n}-u_{j-1}^{n}, f_{j}^{n}\right)_{B_{2}^{1}}+\frac{1}{2}\left\|u_{j-1}^{n}\right\|^{2}+h_{n}\left(\delta u_{j}^{n}, h_{n} \sum_{i=1}^{j-1} k_{j i}^{n} g_{i}^{n}\right)_{B_{2}^{1}} .
\end{aligned}
$$

Ignoring the first two terms in the left hand side, we have

$$
\begin{aligned}
& \left\|u_{j}^{n}\right\|^{2} \leq 2 h_{n}\left\|f_{j}^{n}\right\|_{B_{2}^{1}}\left\|\delta u_{j}^{n}\right\|_{B_{2}^{1}}+\left\|u_{j-1}^{n}\right\|^{2}+h_{n}^{2} \sum_{i=1}^{j-1}\left|k_{j i}^{n}\right|\left\|g_{i}^{n}\right\|_{B_{2}^{1}}\left\|\delta u_{j}^{n}\right\|_{B_{2}^{1}} \\
& \leq C h_{n}+\left\|u_{j-1}^{n}\right\|^{2}+C h_{n}^{2} \sum_{i=1}^{j-1}\left\|u_{i}^{n}\right\|_{B_{2}^{1}} .
\end{aligned}
$$

Repeating the above inequality and using the similar arguments as above we get the required estimate (3.23). This completes the proof of the lemma.

Remark 3.3 From Lemma 3.2 it follows that the function $U^{n}$ is Lipschitz continuous on $[0, T]$. The sequence $\left\{U^{n}(t)\right\}$ and $\left\{X^{n}(t)\right\}$ are uniformly bounded

$\left\|U^{n}(t)\right\| \leq C, \quad\left\|X^{n}(t)\right\| \leq C, \quad \forall t \in J$,

$X^{n}(t)-U^{n}(t) \rightarrow 0$ as $n \rightarrow \infty$ uniformly on $J$.

Lemma 3.4 There exists a function $u \in L^{2}(J ; V) \cap C\left(J ; B_{2}^{1}(0,1)\right)$ with $\frac{d u}{d t} \in$ $L^{2}\left(J ; B_{2}^{1}(0,1)\right)$ and subsequence $\left\{U^{n_{p}}\right\}_{p}$ of $\left\{U^{n}\right\}_{n}$ and $\left\{X^{n_{p}}\right\}_{p}$ of $\left\{X^{n}\right\}_{n}$ such that

$$
\begin{aligned}
& U^{n_{p}}-u \text { and } \quad X^{n_{p}}-u, \quad \text { in } L^{2}(J ; V), \\
& \frac{d U^{n_{p}}}{d t}-\frac{d u}{d t} \text { in } L^{2}\left(J ; B_{2}^{1}(0,1)\right)
\end{aligned}
$$

where $" \rightarrow$ " denotes the weak convergence.

Proof. Remark (3.3) implies that the Rothe sequence $\left\{U^{n}(t)\right\}$ and the sequence $\left\{X^{n}(t)\right\}$ of step functions are bounded in the space $L^{2}(J ; V)$. Since these are in a Hilbert space, subsequence $\left\{U^{n_{p}}(t)\right\}$ and $\left\{X^{n_{p}}(t)\right\}$ can be found weakly convergent in the space to abstract functions $u, x \in L^{2}(J ; V)$, respectively,

$$
U^{n_{p}}-u \quad \text { and } \quad X^{n_{p}} \rightarrow x \quad \text { in } L^{2}(J ; V) .
$$

To show that $u=x$ we have to show, that 


$$
\left(v, u-X^{n_{p}}\right)_{L^{2}(J ; V)} \rightarrow 0, \quad \forall v \in L^{2}(J ; V)
$$

The convergence in (3.36) follows from Remark 3.3 and the following inequality

$$
\left|\left(v, u-X^{n_{p}}\right)_{L^{2}(J ; V)}\right| \leq\left|\left(v, u-U^{n_{p}}\right)_{L^{2}(J ; V)}\right|+\|v\|_{L^{2}(J ; V)}\left\|U^{n_{p}}-X^{n_{p}}\right\|_{L^{2}(J ; V)}(3
$$

Passing to the limit in (3.37) we have $u=x$. We define a sequence of abstract functions $Y^{n}(t): J \rightarrow L^{2}(0,1)$ by

$$
Y^{n}(0)=\delta u_{1}^{n}, \quad Y^{n}(t)=\delta u_{j}^{n}, \quad t \in\left(t_{j-1}^{n}, t_{j}^{n}\right], j=1,2, \ldots \ldots, n .
$$

From Lemma 3.2 it follows that the sequence $\left\{Y^{n}(t)\right\}$ is bounded in the space $L^{2}\left(J ; B_{2}^{1}(0,1)\right)$, a subsequence $\left\{Y^{n_{p}}(t)\right\}$ can be found converging weakly to a function $y \in L^{2}\left(J ; B_{2}^{1}(0,1)\right)$,

$Y^{n_{p}}-y$ in $\quad L^{2}\left(J ; B_{2}^{1}(0,1)\right)$

Thus the integral

$$
\int_{0}^{t} y(s) d s=w(t)
$$

exists it follows that in the space $L^{2}\left(J ; B_{2}^{1}(0,1)\right)$ we have

$U^{n_{p}}(t)-u_{0}=\int_{0}^{t} Y^{n_{p}}(s) d s$.

From (3.35) and (3.38) we have $w=u$ in $L^{2}\left(J ; B_{2}^{1}(0,1)\right)$ and $\frac{d u}{d t}(t)=y(t)$ in $L^{2}\left(J ; B_{2}^{1}(0,1)\right)$. Since $\frac{d U^{n_{p}}}{d t}=Y^{n_{p}}$ it follows that

$\frac{d U^{n_{p}}}{d t} \rightarrow \frac{d u}{d t} \quad$ in $\quad L^{2}\left(J ; B_{2}^{1}(0,1)\right)$

This completes the proof of the lemma.

Remark 3.5 The function $u(t)$ from Lemma 3.4 possesses the following properties:

(a) Since $u \in L^{2}(J ; \boldsymbol{V})$ we have for almost all $t \in J, u \in \boldsymbol{V}$. The integral boundary condition (1.4) of the problem satisfied. The initial condition is fulfilled in the sense of (3.39).

(b) $u$ is strongly differentiable a.e. in $J$ and $\frac{d u}{d t} \in L^{2}\left(J ; B_{2}^{1}(0,1)\right)$;

(c) $U^{n_{p}}(t)$ and $X^{n_{p}}(t) \rightarrow u(t)$ in $L^{2}\left(J ; B_{2}^{1}(0,1)\right)$ for all $t \in J$; 
(d) $\frac{d U^{n} p(t)}{d t} \rightarrow \frac{d u}{d t}$ in $L^{2}\left(J ; B_{2}^{1}(0,1)\right)$.

Definition 3.6 Further, we define the sequence $\left\{f^{n}(t)\right\}$ and $\left\{K^{n}(t)\right\}$ of step functions from $J$ into $\boldsymbol{H}^{2}$ by

$$
f^{n}(0)=f_{0}, f^{n}(t)=f\left(t_{j}^{n}-u_{0}\left(t_{j}^{n}-\tau\right)\right), \quad t \in\left(t_{j-1}^{n}, t_{j}^{n}\right], \quad 1 \leq j \leq n .
$$

And

$K^{n}(0)=h_{n} k_{10}^{n} g_{1}, K^{n}(t)=h_{n} \sum_{i=1}^{j-1} k_{j i}^{n} g_{i}, \quad t \in\left(t_{j-1}^{n}, t_{j}^{n}\right], \quad 1 \leq i, j \leq n$.

Integral identity (3.20) may be rewritten as

$$
\left(v(t), \frac{d^{-}}{d t} U^{n}(t)\right)_{B_{2}^{1}}+\left(v(t), X^{n}(t)\right)=\left(v(t), f^{n}(t)\right)_{B_{2}^{1}}+\left(v(t), K^{n}(t)\right)_{B_{2}^{1}},
$$

for all $v \in \boldsymbol{V}$ and a.e. $t \in(0, T]$, where $\frac{d^{-}}{d t}$ denote the left hand derivative.

Lemma 3.7 Let $\left\{K^{n}(t)\right\}$ be the sequence of functions defined by (3.42) and

$$
K(u)(t)=\int_{0}^{t} k(t-s) g(s, u(s)) d s,
$$

where $u$ is determined from Lemma 3.4. Then

$$
K^{n_{p}}(t) \rightarrow K(u)(t)
$$

uniformly on $[0, T]$ as $p \rightarrow \infty$.

Proof. Let $K(\varnothing)(t)$ denote the integral

$$
K(\varnothing)(t)=\int_{0}^{t} k(t-s) g(s, \emptyset(s)) d s
$$

where $\varnothing: J \rightarrow X$ is a piecewise continuous function. We first show that $K\left(X^{n_{p}}\right)(t) \rightarrow K(u)(t)$, uniformly on $[0, T]$ as $p \rightarrow \infty$.

$$
\left\|K(u)(t)-K\left(X^{n_{p}}\right)(t)\right\|_{B_{2}^{1}} \leq k_{T} \int_{0}^{t}\left\|g(s, u(s))-g\left(s, X^{n_{p}}(s)\right)\right\|_{B_{2}^{1}} d s,
$$


hence $K\left(X^{n_{p}}\right)(t) \rightarrow K(u)(t)$, uniformly on $[0, T]$ as $p \rightarrow \infty$. We now show that $K^{n_{p}}(t)-K\left(X^{n_{p}}\right)(t) \rightarrow 0$ as $p \rightarrow \infty$ uniformly on $J$. For $t \in\left(t_{j-1}^{n_{p}}, t_{j}^{n_{p}}\right]$, we have

$$
\begin{aligned}
K^{n_{p}}(t)-K\left(X^{n_{p}}\right)(t)=h_{n} \sum_{i=0}^{j-1} k_{j i}^{n_{p}} g_{i}-\int_{0}^{t} k(t-s) g\left(s, X^{n_{p}}(s)\right) d s \\
=\sum_{i=1}^{j-1} \int_{t_{i-1}^{n_{p}}}^{t_{i}^{n_{p}}}\left[k_{j i}^{n_{p}} g\left(t_{i}, u_{i}\right)-k(t-s) g\left(s, u_{i}\right)\right] d s \\
+h_{n} k\left(t_{j}^{n_{p}}-t_{0}^{n_{p}}\right) g\left(t_{0}, u_{0}\right)-\int_{t_{j-1}^{n_{p}}}^{t} k(t-s) g\left(s, X^{n_{p}}(s)\right) d s .
\end{aligned}
$$

Remark 3.3 and assumptions (D1) and (D4) imply that the last two terms on the right hand side tend to zero strongly and uniformly on $J$ as $p \rightarrow \infty$. Since $k$ and $g$ are continuous, for every $\epsilon>0$ we can choose a positive integer $n$ sufficiently large such that for $t \in\left(t_{j-1}, t_{j}\right]$, and $s \in\left(t_{i-1}, t_{i}\right]$, we have

$$
\left\|k_{j i}^{n_{p}} g\left(t_{i}, u_{i}\right)-k(t-s) g\left(s, u_{i}\right)\right\|_{B_{2}^{1}} \leq \frac{\epsilon}{T}
$$

Therefore

$$
\left\|\sum_{i=1}^{j-1} \int_{t_{i-1}^{n_{p}}}^{t_{i}^{n_{p}}}\left[k_{j i}^{n_{p}} g\left(t_{i}, u_{i}\right)-k(t-s) g\left(s, u_{i}\right)\right] d s\right\|_{B_{2}^{1}} \leq j h_{n} \frac{\epsilon}{T}<\epsilon
$$

which show that $K^{n_{p}}(t)-K\left(X^{n_{p}}\right)(t) \rightarrow 0$ as $p \rightarrow \infty$, uniformly on $[0, T]$. This completes the proof of the lemma.

\section{Proof of the Theorem 2.2}

Proof. In view of Remark 3.5, it remains to show that integral identity (2.10) is satisfied by $u(t)$. We consider the integral identity (3.43) written for $n_{p}$ :

$\left(v, \frac{d^{-}}{d t} U^{n_{p}}(t)\right)_{B_{2}^{1}}+\left(v, X^{n_{p}}(t)\right)=\left(v, f^{n_{p}}(t)\right)_{B_{2}^{1}}+\left(v, K^{n_{p}}(t)\right)_{B_{2}^{1}}, \quad \forall v \in V .(4.44)$

Integrating (4.44) between 0 and $T$. We obtain 


$$
\begin{aligned}
\int_{0}^{T}(v & \left.\frac{d^{-}}{d t} U^{n_{p}}(t)\right)_{B_{2}^{1}} d t+\int_{0}^{T}\left(v, X^{n_{p}}(t)\right) d t \\
& =\int_{0}^{T}\left(v, f^{n_{p}}(t)\right)_{B_{2}^{1}} d t+\int_{0}^{T}\left(v, K^{n_{p}}(t)\right)_{B_{2}^{1}} d t, \quad \forall v \in L^{2}(J, V) .
\end{aligned}
$$

Now, as $n_{p} \rightarrow \infty$, we have

$\frac{d^{-}}{d t} U^{n_{p}}(t) \rightarrow \frac{d u}{d t}(t)$ in $L^{2}\left(J ; B_{2}^{1}(0,1)\right)$,

and

$\int_{0}^{T}\left(v, \frac{d^{-}}{d t} U^{n_{p}}(t)\right)_{B_{2}^{1}} d t \rightarrow \int_{0}^{T}\left(v, \frac{d^{-}}{d t} u(t)\right)_{B_{2}^{1}} d t, \quad$ for $n_{p} \rightarrow \infty$

Further, for a fixed $v \in L^{2}(J ; V)$ the linear functional $\left(v, X^{n}(t)\right)$ is uniformly bounded on $L^{2}(J ; V)$, and from the convergence result in Lemma 3.4, we have

$$
\int_{0}^{T}\left(v, X^{n_{p}}(t)\right) d t \rightarrow \int_{0}^{T}(v, u(t)) d t, \quad \text { for } n_{p} \rightarrow \infty
$$

Now using Lemma 3.7, (4.47) and (4.48), passing to the limit in (4.45) as $n_{p} \rightarrow \infty$, we have

$$
\int_{0}^{T}\left(v, \frac{d u(t)}{d t}\right)_{B_{2}^{1}} d t+\int_{0}^{T}(v, u(t)) d t=\int_{0}^{T}(v, f(t, u(t-\tau))+K(u)(t))_{B_{2}^{1}} d t
$$

for all $v \in L^{2}(J ; V)$.

Uniqueness: Let two weak solutions $u_{1}(t), u_{2}(t)$ exist. Denote their difference by $u(t)=u_{2}(t)-u_{1}(t)$. Then $u(t)$ satisfies

$$
\begin{aligned}
\int_{0}^{T}\left(v(t), \frac{d u(t)}{d t}\right)_{B_{2}^{1}} d t & +\int_{0}^{T}(v(t), u(t)) d t \\
& =\int_{0}^{T}\left(v(t), \int_{0}^{t} k(t-s)\left[g\left(s, u_{2}(s)\right)-g\left(s, u_{1}(s)\right)\right] d s\right)_{B_{2}^{1}} d t,
\end{aligned}
$$

for all $v \in L^{2}(J ; V)$. We have to show that $u \equiv 0$. Let us divide the interval $J$ into a finite number of subintervals of lengths $l$, with $l$ satisfying

$$
k_{T} l^{2} \int_{0}^{T} L(s) d s \leq \frac{1}{2} \text { where } k_{T}=\max \{\mid k(t), t \in J\} .
$$

The function $\|u(t)\|$ is continuous in $J$ and in $J_{l}=[0, l]$. Consequently, $\|u(t)\|$ attains its maximum on certain point $t_{1} \in J_{l}$,

$$
\max _{t \in[0, l]}\|u(t)\|=\left\|u\left(t_{1}\right)\right\| \text {. }
$$


Take

$$
v(t)=\left\{\begin{array}{cl}
u(t), & \text { for } t \in\left[0, t_{1}\right] \\
0, & \text { for } t \in\left(t_{1}, T\right]
\end{array}\right.
$$

as a test function in (4.49), we obtain

$$
\begin{aligned}
\int_{0}^{t_{1}}\left(u(t), \frac{d u(t)}{d t}\right)_{B_{2}^{1}} d t+\int_{0}^{t_{1}}\|u(t)\|^{2} d t \\
\quad=\int_{0}^{t_{1}}\left(u(t), \int_{0}^{t} k(t-s)\left[g\left(s, u_{2}(s)\right)-g\left(s, u_{1}(s)\right)\right] d s\right)_{B_{2}^{1}} d t .
\end{aligned}
$$

According to assumption (D2), we have

$$
\frac{1}{2}\left\|u\left(t_{1}\right)\right\|^{2}=\left\|u\left(t_{1}\right)\right\|^{2} k_{T} l^{2} \int_{0}^{T} L(s) d s \leq \frac{1}{4}\left\|u\left(t_{1}\right)\right\|^{2},
$$

and so $u(t)=0$ on $[0, l]$. Similar argument are repeated to show that $u \equiv 0$ on $[i l,(i+1) l], i=1,2, \ldots$. which yields $u \equiv 0$ on $J$.

Continuous dependence: Let $u_{1}$ and $u_{2}$ be two weak solutions of (1.1)-(1.4), corresponding to $\left(u_{1}^{0}, f_{1}\right)$ and $\left(u_{2}^{0}, f_{2}\right)$, respectively, and the initial data satisfy the given assumptions, from (2.10), putting $u=u_{1}-u_{2}$, and the assumption on $g(t, u)$ we have

$$
\begin{aligned}
& \frac{d}{d t}\|u(t)\|_{B_{2}^{1}}^{2}+2\|u(t)\|^{2} \\
& =2\left(f_{1}(t, u(t-\tau))-f_{2}(t, u(t-\tau)), u(t)\right)_{B_{2}^{1}}+2\left(K\left(u_{1}\right)(t)-\right. \\
& \left.K\left(u_{2}\right)(t), u(t)\right)_{B_{2}^{1}} \\
& \leq\left\|f_{1}(t, u(t-\tau))-f_{2}(t, u(t-\tau))\right\|_{B_{2}^{1}}^{2}+\|u(t)\|_{B_{2}^{1}}^{2}+C_{T}\|u\|_{C\left(J_{t} ; B_{2}^{1}\right)}^{2},
\end{aligned}
$$

where $J_{t}=[0, t]$ and $C_{T}=k_{T} \int_{0}^{T} L(s) d s$.

Integrating over $(0, s)$ for $0 \leq s \leq t \leq T$ and using the fact that $u_{i}(0)=u_{i}^{0}$, for $i=1,2$, we get

$$
\begin{aligned}
& \|u(t)\|_{B_{2}^{1}}^{2}+2 \int_{0}^{t}\|u(s)\|^{2} d s \\
& \leq\left\|u_{1}^{0}-u_{2}^{0}\right\|_{B_{2}^{1}}^{2}+\int_{0}^{t}\left\|\tilde{f}_{1}(s)-\tilde{f}_{2}(s)\right\|_{B_{2}^{1}}^{2} d s+C_{T} \int_{0}^{t}\|u\|_{C\left(J_{s} ; B_{2}^{1}\right)}^{2} d s \\
& \quad+\int_{0}^{t}\|u(s)\|_{B_{2}^{1}}^{2} d s+\int_{0}^{t}\|u(s)\|^{2} d s .
\end{aligned}
$$


Taking the supremum and applying Gronwall's inequality we obtain

$$
\begin{aligned}
& \left\|u_{1}-u_{2}\right\|_{C\left(J_{t} ; B_{2}^{1}\right)}^{2}+2 \int_{0}^{t}\left\|u_{1}-u_{2}\right\|_{C\left(J_{s} ; L^{2}\right)}^{2} d s \\
& \quad \leq\left(\left\|u_{1}^{0}-u_{2}^{0}\right\|_{B_{2}^{1}}^{2}+C_{T}\left\|u_{1}-u_{2}\right\|_{C\left(J_{t} ; B_{2}^{1}\right)}^{2}+\int_{0}^{t}\left\|\tilde{f}_{1}-\tilde{f}_{2}\right\|_{C\left(J_{s} ; B_{2}^{1}\right)}^{2} d s\right) \exp \{t\} .
\end{aligned}
$$

This shows the continuous dependence of the solution on the initial data. This completes the proof of the theorem.

Remark 4.1 Uniqueness of the weak solution $u(t)$ implies that not only the sequence $\left\{U^{n_{p}}(t)\right\}$ converges weakly to $u(t)$, but the whole sequence $\left\{U^{n}(t)\right\}$ converges weakly to $u(t)$. This can be proved as follows. Suppose that $\left\{U^{n}(t)\right\}$ does not converge weakly to $u(t)$ in $L^{2}(J, V)$. Then there exists at least one function $v \in L^{2}(J, V)$ and an $\epsilon>0$ such that

$$
\left|\int_{0}^{T}\left(v(t), u(t)-U^{n}(t)\right) d t\right| \geq \epsilon
$$

for infinitely many values of $n$. Let $\left\{U^{n_{k}}(t)\right\}$ be a sequence satisfying (4.50). This sequence, being a subsequence of $\left\{U^{n}(t)\right\}$ is bounded in $L^{2}(J ; V)$. Consequently a subsequence $\left\{U^{n_{k}}\right\}$ can be found converging weakly in $L^{2}(J ; V)$ to a function $\xi \in L^{2}(J ; V)$. This function have all the properties of the solution of the problem, thus it is also the weak solution of the problem (1.1)-(1.4) and $u \neq \xi$ in $L^{2}(J ; V)$. This contradicts the fact that the problem has a unique weak solution.

Example 4.2 Consider the initial and nonlocal boundary value problem for the heat equation

$$
\begin{aligned}
& \frac{\partial u}{\partial t}(x, t)-\frac{\partial^{2} u}{\partial t^{2}}(x, t)=1-\pi^{2} t+\frac{\pi^{4} t^{2}}{2}-e^{-\pi^{2} t}+e^{\pi^{2}(t-\tau)} \cos \pi x-u(x, t-\tau) \\
& +\pi^{4} \int_{0}^{t}(t-s)|1-u(x, s)| d s, \quad t>0, \quad x \in(0,1) \\
& u(x, 0)=\cos \pi x, \quad x \in(0,1), \\
& \frac{\partial u}{\partial x}(0, t)=0, \quad t \geq 0, \\
& \int_{0}^{1} u(x, t) d x=0, t \geq 0 .
\end{aligned}
$$

It may be seen that all the assumption of theorem 2.2 are satisfied for the functions appearing in the above example. Hence this problem has a unique solution. We may verify that $u(x, t)=e^{-\pi^{2} t} \cos \pi x$ is the solution of (4.51)(4.54). 


\section{References}

[1] D. Bahuguna, J. Dabas, Existence and uniqueness of solution to a semilinear partial delay differential equation with an integral condition, Nonlinear Dyn. Syst. Theory 8 (1) (2008) 7-19.

[2] D. Bahuguna, S. Abbas, J. Dabas, Partial functional differential with an integral condition and application to population dynamics, Nonlinear anal., TMA 69 (3-4) (2008) 2623-2635.

[3] D. Dao-Quing, H. Yu, Remarks on heat equation with integral boundary conditions, Nonlinear Analysis, TMA. 67 (2) (2007) 468-475.

[4] D. Bahuguna and V. Raghavendra, Rothe's method to parabolic integrodifferential equations via abstract integro-differential equations, Appl. Anal. 33 (3-4) (1989) 153-167

[5] J.R. Cannon, The solution of the heat equation subject to the specification of energy, Quart. Appl. Math. 21 (1963) 155-160.

[6] J.R. Cannon, J.V.D. Hoek, Diffusion subject to the specification of mass, J. Math. Anal. Appl. 115 (1986) 517-529.

[7] J.R. Cannon, Y. Lin, A Galerkin procedure for diffusion equation subject to specification of mass, SIAM J. Numer. Anal. 24 (1987) 499-515.

[8] K. Rektorys, The method of discretization in time and partial differential equations, Reidel, Dordrecht,Bostan,Londan (1982).

[9] L.I. Kamynin, A boundary value problem in the theory of the heat conduction with nonclassical boundary condition, Z. Vychisl. Mat. Fiz. 6 (1964) 1006-1024.

[10] L.S. Pulkina, A nonlocal problem with integral conditions for hyperbolic equations, Electronic J. of Differential Eqns 45 (1999) 1-6.

[11] N.I. Ionkin, Solutions of boundary value problem in heat conduction theory with nonlocal boundary conditions, Differents. Urav. 13 (1977) 294-304.

[12] N. Merazga, A. Bouziani, on a time-discretization method for a semilinear heat equation with purely integral conditions in a nonclassical function space, Nonlinear Anal, TMA. 66 (2007) 604-623.

[13] N. Merazga, A. Bouziani, Rothe time-discretization method for a nonlocal problem arising in thermoelasticity, J. Appl. Math. Stoch. Anal. 1 (2005) 1328.

[14] N. Merazga, A. Bouziani, Rothe method for mixed problem with an integral condition for the two dimensional diffusion equations, Abstract and applied analysis. 16 (2003) 899-922.

[15] S.A. Beilin, Existence of solutions for one dimensional wave equations with nonlocal conditions, Electronic J. of Differential Eqns 76 (2001) 1-8. 